\title{
A study on changes of the resting-state brain function network in patients with amnestic mild cognitive impairment
}

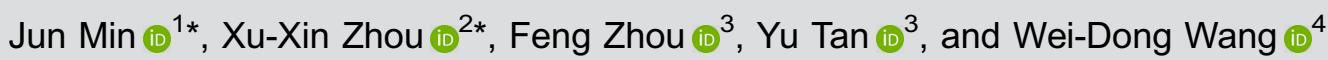 \\ ${ }^{1}$ Department of Rehabilitation, Third Affiliated Hospital of Nanchang University, Jiangxi, China \\ ${ }^{2}$ Department of Biomedical Engineering, School of Measuring and Optical Engineering, Nanchang Hangkong University, \\ Nanchang, China \\ ${ }^{3}$ Department of Neurology, Third Affiliated Hospital of Nanchang University, Nanchang, China \\ ${ }^{4}$ Department of Neurology, Shenzhen Hospital of Southern Medical University, Shenzhen, China
}

\begin{abstract}
This study aimed to explore the structural and functional characteristics of the neural network of resting-state brain activities in patients with amnestic mild cognitive impairment (aMCl) by functional magnetic resonance imaging (fMRI) technology. Resting state $\mathrm{fMRI}$ scanning was performed on 10 clinically diagnosed $\mathrm{aMCl}$ patients and 10 healthy volunteers, and the difference in the resting-state brain activities between aMCI patients and healthy volunteers was compared using the brain function network regional homogeneity $(\mathrm{ReHo})$ analysis method. Results of the $\mathrm{ReHo}$ analysis of aMCl patients and healthy volunteers revealed that the ReHo value significantly increased in the posterior cingulate gyrus region, medial frontal lobe, medial cortex of the prefrontal lobe, and part of the parietal lobe. Compared with the normal elderlies, ReHo decreased in aMCl patients in the left temporal lobe (middle temporal gyrus and inferior temporal gyrus), left parahippocampal gyrus, occipital lobe, lingual gyrus, precuneus, and other regions while ReHo increased in regions of the right frontal lobe (inferior frontal gyrus), left superior temporal gyrus, precentral gyrus (frontal lobe), right thalamus, left fusiform gyrus, and other regions. In the resting state, there may be regional abnormalities in brain functional areas in aMCl patients, which may be associated with cognitive impairment.
\end{abstract}

Key words: Amnestic; Mild cognitive impairment; Function network; Resting state; Functional magnetic resonance imaging

\section{Introduction}

Alzheimer's disease (AD) is the most common cause of senile dementia. It is characterized by progressive memory loss, which gradually impacts a patient's cognition and non-cognitive functions. It has been reported that approximately $10-15 \%$ of patients with mild cognitive impairment $(\mathrm{MCl})$ develops into $A D$ per year (1). The clinical manifestations and prognosis of $\mathrm{MCl}$ patients are heterogeneous. $\mathrm{MCl}$ patients not only exhibit memory impairment, but also exhibit mild cognitive impairments of attention, executive ability, and other cognitive functions. Therefore, Petersen et al. (2) proposed the concept of amnestic $\mathrm{MCl}(\mathrm{aMCl})$ based on clinical evaluations. Episodic memory disorder is the outstanding manifestation of $\mathrm{aMCl}$, and the annual conversion rate of $\mathrm{aMCl}$ to $\mathrm{AD}$ is 10 times that of the incidence of $A D$ in the normal population. Therefore, aMCl is considered the precursor phase of $A D$; if early diagnosis and treatment are performed at this stage, patients' quality of life will be improved.

Brain functional magnetic resonance imaging (fMRI) is mainly performed based on the principle of blood oxygenation level dependent (BOLD) contrast enhancement. Before $\mathrm{MCl}$ progresses into $\mathrm{AD}, \mathrm{BOLD}-\mathrm{fMRI}$ is able to detect changes in brain functions, and it is an important technique for studying the neural mechanism of $\mathrm{MCl}$ (3). For example, Qi, et al. (4) found that diabetes mellitus type 2 (T2DM) patients with aMCl showed abnormal functional connectivity patterns and decreased white matter integrity, making fMRI a potential diagnostic tool for early detection of $A D$ in elderly T2DM patients. Another study used fMRI to evaluate the resting-state brain function. The authors demonstrated that changes in functional brain networks involved in cognition, such as episodic memory and visual

Correspondence: Wei-Dong Wang: <wdwang_621@163.com>

${ }^{*}$ These authors contributed equally to this study.

Received October 16, 2018 | Accepted February 12, 2019 
cognition in aMCl, were altered, and provided new insight into understanding the important subtype of aMCl (5).

In the present study, resting fMRI technology was used to compare the ReHo between normal elderlies and $\mathrm{aMCl}$ patients, aiming to explore the homogeneity and differences of brain functional activities in the resting state in both populations, and to provide a candidate region of interest for subsequent study on brain functional connectivity, as well as a basis for the early diagnosis of $\mathrm{aMCl}$.

\section{Material and Methods}

\section{Research subjects}

aMCl group. Ten patients who were diagnosed with $\mathrm{aMCl}$ by neuropsychological testing and imaging examination at the Third Affiliated Hospital of Nanchang University from September 2010 to December 2011 were selected. According to the diagnostic criteria of $\mathrm{aMCl}$ proposed by Petersen et al. in 2001 (1), inclusion criteria were as follows: 1 ) patients between $65-75$ years old with Han nationality and right-handed; 2) patients whose chief complaint was memory loss, and this symptom had been lasting for more than six months; 3 ) patients with normal general cognitive function and Mini Mental State Examination (MMSE) scores between 24-28 points; 4) patients whose ability of daily life was preserved and had ability of daily living (ADL) scores of $<26$ points; 5 ) cognitive disorders did not meet the diagnosis of dementia, and the clinical dementia rating (CDR) scale score was 0.5 points. Exclusion criteria were as follows: 1) patients with a Hachinski ischemic score of $>4$ points and a history of acute cerebral vascular disease in the past three months; 2) patients with active epilepsy; 3) patients with Hamilton Depression Scale (Hamilton) scores of $>12$ points and had a history of mental illness; 4) patients with the presence of liver and kidney dysfunction that affected cognitive function, deficiencies of folic acid and vitamin B12, abuse of drugs and alcohol, and severe infection; 5) patients with advanced, serious, or other unstable diseases, which affect the assessment of brain function or cognitive function. A total of 10 patients were enrolled including five males and five females, with an average age of $69.80 \pm$ 2.658 years. The average years of education was $13.20 \pm$ 1.135 , and the MMSE score was $25.90 \pm 0.738$ points.

Normal control group. Ten normal elderlies with matched age, gender, and education level were enrolled in this study and underwent physical examinations at the same period. The inclusion criteria were: 1) subjects between 65 to 75 years old with Han nationality and righthanded, who had good overall health conditions and had no nervous system disease; 2) no memory complaints and clinical manifestations; 3 ) normal overall cognitive function and MMSE scores of $>27$ points; 4) ADL scale scores of $<26$ points; 5 ) no cognitive impairment and a CDR scale score of zero point. Five males and five females were enrolled with an average age of $69.90 \pm 2.601$ years,
$13.60 \pm 1.713$ average educational years, and an average MMSE score of $29.30 \pm 0.823$ points.

There was no significant difference in age, educational years, or gender between the aMCl group and the control group $(\mathrm{P}>0.05)$. However, there was a significant difference in MMSE scores between these two groups $(P<0.001)$. This research was approved by the Medical Ethics Committee of Nanchang University. All subjects and their families were informed of the research content, were willing to cooperate with the trial, and provided written informed consent.

\section{Research methods}

MR scanning. A GE-3.0T superconducting magnetic resonance scanner (USA) was used. During the whole process, subjects were instructed to remain in the resting state, keep awake, take the supine position, close their eyes, try to relax, and refrain from moving. The head was fixed, and the active and passive relationship between the head and other parts were maximally reduced. At the same time, any brain activity for thinking by the subjects was avoided. The resting state scan parameters were: gradient echo planar imaging sequence, repetition time $(\mathrm{TR})=2,000 \mathrm{~ms}$, echo delay time $(\mathrm{TE})=30 \mathrm{~ms}$, flip angle= $90^{\circ}$, field of view $(F O V)=25.6$, array $=64 \times 64$, slice thickness $=3 \mathrm{~mm}$, slice gap $=1.0 \mathrm{~mm}$, number of slices $=32$, and total time $=6 \mathrm{~min}$ and $40 \mathrm{~s}$.

Data processing. The statistical parametric mapping software running on the 2010 Matlab (MathWorks, USA) was used. The format of the acquired imaging data was transformed using the MRIcro software package (http:// www.mricro.com), alignments at the dimensions of space and time and the correction of head movements were performed using SPM8 and DPARSF software (Institute of Psychology, Chinese Academy of Sciences, China), and head motion parameters during the scanning were obtained at the same time. The first 10 images were excluded, considering that a certain amount of time was needed to make the magnetic field steady, as well as to allow the subjects to adapt to the environment. The remaining 190 images were included in the analysis processes. These images met the criteria, and were normalized using the EPI template, and re-sampled to $3 \times 3 \times$ $3 \mathrm{~mm}^{3}$. Spatial smoothing was performed using a 4-mm Gauss kernel with a full width at half maximum to reduce the spatial noise and anatomical differences in the structures of these subjects. Then, the linear drift was removed by removing the six head dynamic parameters using the linear regression method. Next, wave filtering was performed $(0.01<\mathrm{f}<0.08 \mathrm{~Hz})$.

ReHo was analyzed after low frequency wave signals were obtained. The ReHo analytical method is a data analysis based on the calculation of Kendall's coefficient of concordance (6). It is used as an index to measure the homogeneity of time-series changes between adjacent voxels, to analyze the homogeneity of spontaneous brain 
activity using whole brain functional imaging data. The homogenous spontaneous activity in the brain is secondary to nerve activity. This method can be used to measure the homogeneity of local BOLD signals, which reflects the homogeneity of nerve activity in the brain.

The two-sample $t$-test was used for inter-group analysis to detect the difference in ReHo values in brain regions between the aMCl group and control group $(\mathrm{P}<0.05$ indicated a statistically significant difference). The size of the mass was more than 20 voxels (uncorrected). A statistical parametric map was added onto the glass map, and the regions with significant differences in these two groups were described using the known neuroanatomical markers.

\section{Results}

In the resting state, ReHo values were significantly elevated in the posterior cingulate cortex, medial frontal lobe, inner side cortex of the prefrontal lobe, and part of the parietal lobe of normal elderlies and $\mathrm{aMCl}$ patients $(P<0.05$, Figures 1 and 2). Compared with the normal elderlies, ReHo values decreased in aMCl patients in the left temporal lobe (middle temporal gyrus and inferior temporal gyrus), left parahippocampal gyrus, occipital lobe, lingual gyrus, precuneus, and other regions $(P<0.05$; Figure 3, Table 1), while ReHo values increased in the right side frontal lobe (inferior frontal gyrus), left superior temporal gyrus, precentral gyrus (frontal lobe), right thalamus, the left fusiform gyrus, and other regions $(\mathrm{P}<0.05$; Figure 4, Table 2).

\section{Discussion}

$\mathrm{MCl}$ is universally recognized as an intermediate state between normal aging and dementia. Since changes in regional cerebral blood flow and metabolism can occur in the early stage of $A D$, the application of functional imaging

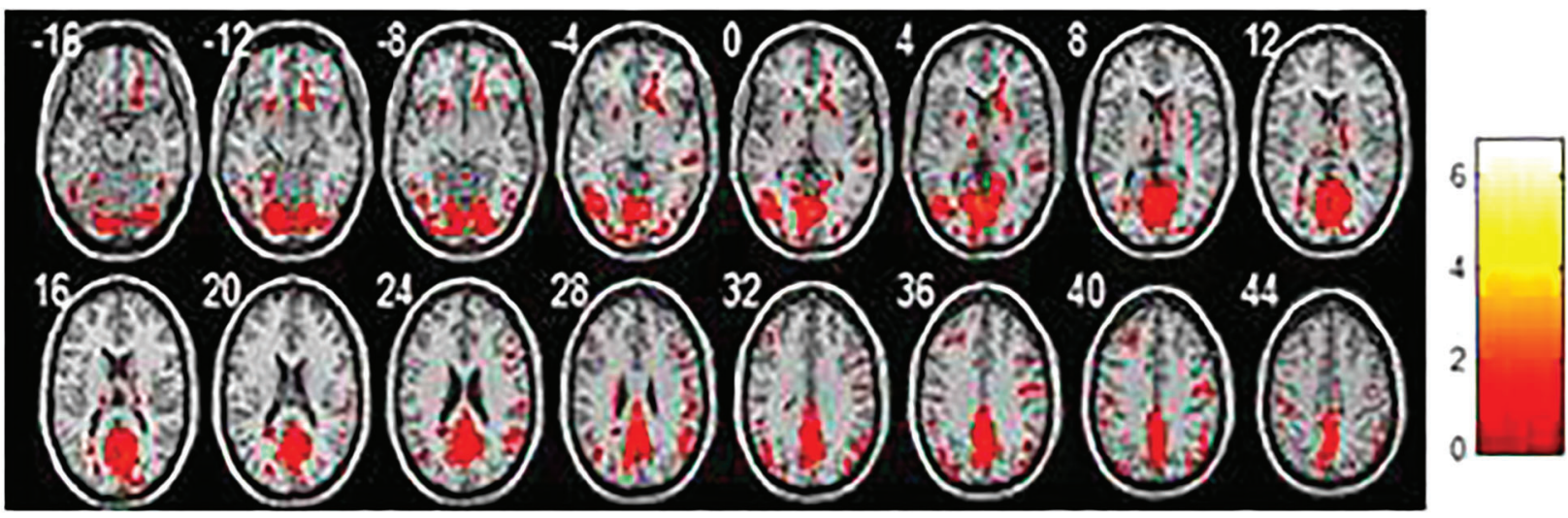

Figure 1. Average regional homogeneity in the normal elderlies. Each image represents a slice, with $4 \mathrm{~mm}$ distance from the other. 0 is the 0 position of space coordinates. The color bar indicates energy intensity.

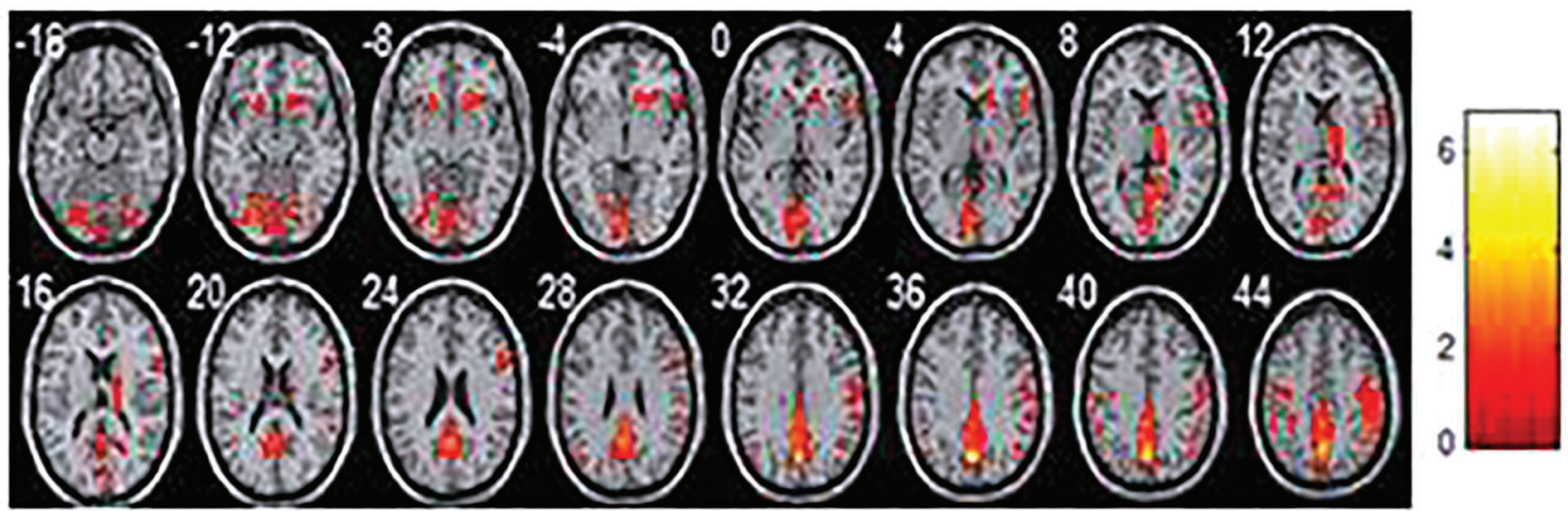

Figure 2. Average regional homogeneity in the amnestic mild cognitive impairment patients. Each image represents a slice, with $4 \mathrm{~mm}$ distance from the other. 0 is the 0 position of space coordinates. The color bar indicates energy intensity. 


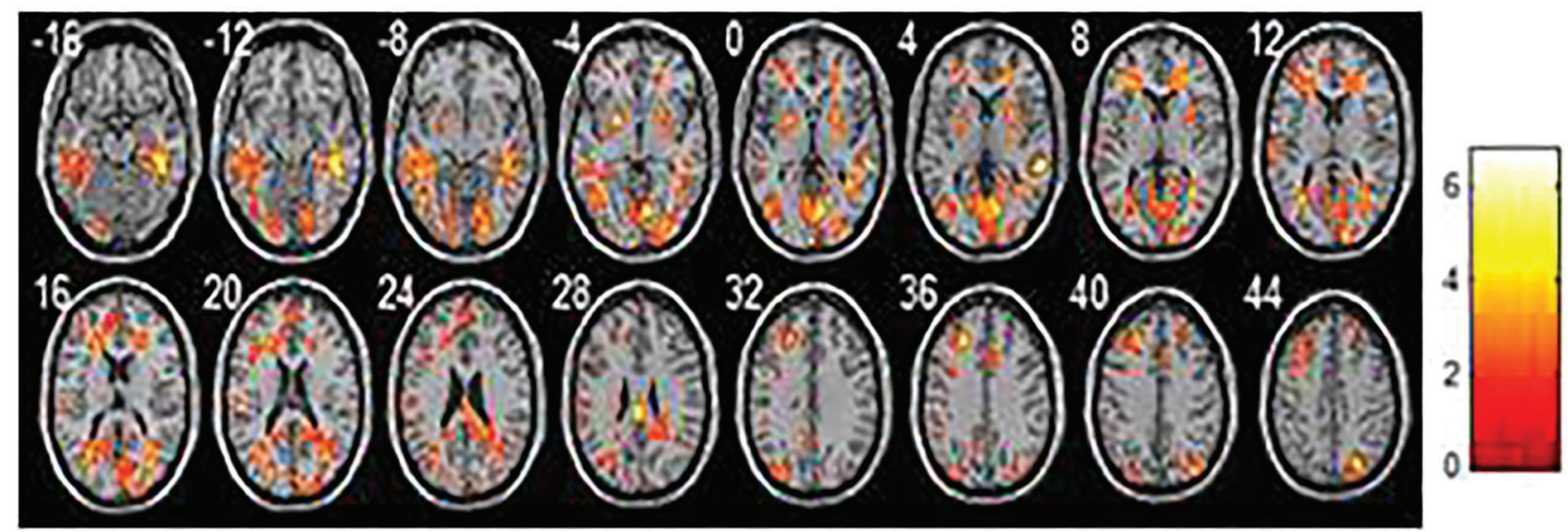

Figure 3. Regions where homogeneity decreased in the amnestic mild cognitive impairment group compared with the normal elderlies. Each image represents a slice, with $4 \mathrm{~mm}$ distance from the other. 0 is the 0 position of space coordinates. The color bar indicates energy intensity.

Table 1. Regions of the brain with decreased regional homogeneity (ReHo) values in amnestic mild cognitive impairment patients compared with the normal elderlies $(P=0.006)$.

\begin{tabular}{lcrrrr}
\hline Brain region & Voxel & \multicolumn{3}{c}{ MNI coordinate } & \multirow{2}{*}{ BA } \\
\cline { 3 - 5 } & & $\mathrm{X}$ & $\mathrm{Y}$ & $\mathrm{Z}$ & \\
\hline Fusiform region & 8 & 21 & -63 & -12 & 19 \\
Frontal lobe & 5 & 15 & 27 & -6 & 47 \\
Middle temporal gyrus & 1 & -57 & -63 & -3 & 37 \\
Inferior frontal gyrus & 8 & 54 & 18 & 0 & 45 \\
Parietal lobes & 32 & 0 & -69 & 36 & 23 \\
\hline
\end{tabular}

technology is helpful for the early diagnosis of AD. Studies have indicated that BOLD-fMRI is a key technique to study the neural mechanism of $\mathrm{MCl}$, since it is able to detect changes in brain functions before $\mathrm{MCl}$ develops into $\mathrm{AD}$ behavior (3).

In the present study, ReHo analysis of the normal elderlies and $\mathrm{aMCl}$ patients revealed that in the resting state, ReHo values were significantly higher in the posterior cingulate gyrus, the inner side of the frontal lobe, the inner side cortex of the prefrontal lobe, and part of the parietal lobe. These brain areas have active functions that reflect the existence of a spontaneous default network in the resting state of the brain. This result is highly consistent with the results from the study of Raichle et al., who found that the exchange of basic information between nerve cells in the brain takes up most of the energy, and a specific task such as memory only accounts for a small part of energy consumption increase. In addition, there are still spontaneous, organized, and continuous brain activities in the resting state. Furthermore, BOLD signals have a high temporal correlation, especially in the inner side cortex of the prefrontal lobe, posterior cortex of the cingulate gyrus, and the outer parietal lobe. Therefore, the concept of a default network was put forward (8).

The present study suggests that the default network is composed of negatively activated brain regions that appear in tasks in a cognitive state, and these brain areas interact with each other to maintain a variety of cognitive activities in the resting state. Research has shown that negative activation is the mechanism of processing the re-allocation of resources (9), assuming that the "resting" state is an organized state of brain activities. When external tasks occur in the brain, these organized processes will be disrupted, related processing resources would be transferred to relevant brain areas associated with the external task, and areas with reduced processing resources would be negatively activated. In addition, Qi et al. (10) demonstrated that abnormality existed in the default mode network of aMCl patients. Moreover, the default-mode network also showed greater abnormalities when compared with high-order visual processing systems in aMCl patients (11).

Compared to the normal elderlies, ReHo values decreased in aMCl patients in the left temporal lobe (middle temporal gyrus and inferior temporal gyrus), 


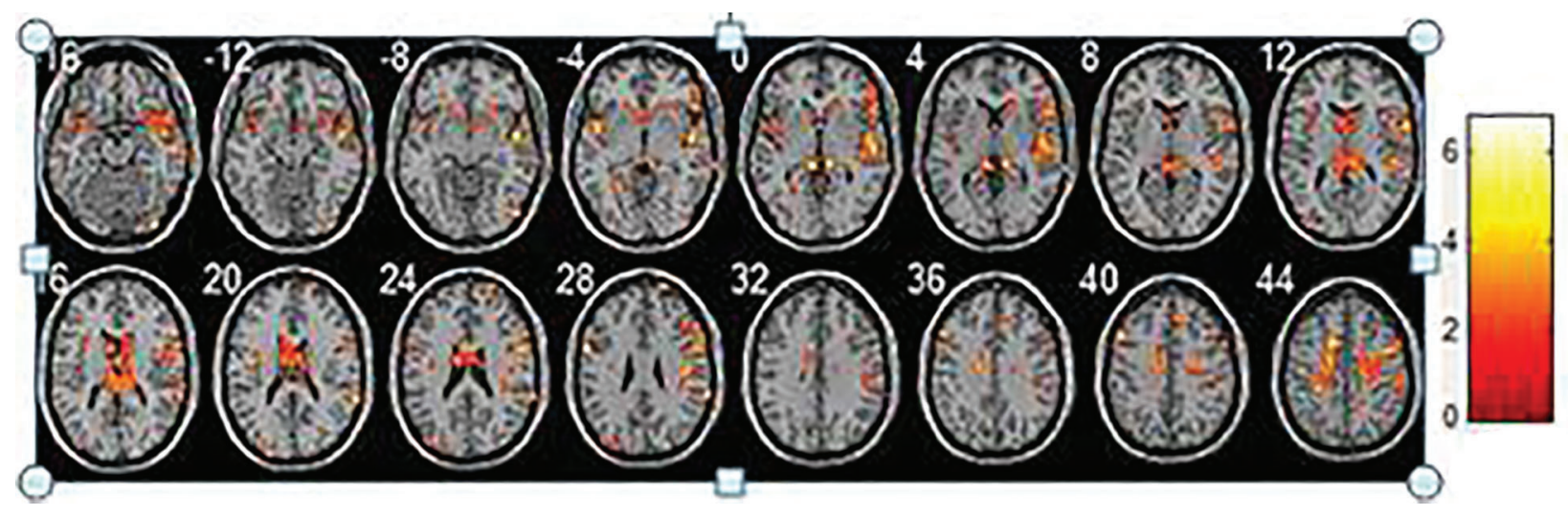

Figure 4. Regions where homogeneity increased in the amnestic mild cognitive impairment groups compared with the normal elderlies. Each image represents a slice, with $4 \mathrm{~mm}$ distance from the other. 0 is the 0 position of space coordinates. The color bar indicates energy intensity.

Table 2. Regions of the brain with increased regional homogeneity in the amnestic mild cognitive impairment $(\mathrm{aMCl})$ group compared with the normal group $(\mathrm{P}=0.008)$.

\begin{tabular}{lrrrrr}
\hline Brain region & Voxel & \multicolumn{3}{c}{ MNI coordinate } & BA \\
\cline { 3 - 5 } & & $\mathrm{X}$ & $\mathrm{Y}$ & $\mathrm{Z}$ & \\
\hline Parietal lobes & 42 & 0 & -69 & 36 & 17 \\
Fusiform region & 11 & 21 & -63 & -12 & 19 \\
Prefrontal lobe & 6 & -18 & 21 & -9 & 47 \\
Inferior frontal gyrus & 28 & 54 & 18 & 0 & 44 \\
\hline
\end{tabular}

left parahippocampal gyrus, occipital lobe, lingual gyrus, precuneus, and other regions. Patients with $A D$ have severe episodic memory impairment due to damage to the storage of information, which is caused by the atrophy of the hippocampus and medial temporal lobe. Episodic memory impairment is the key symptom for the diagnosis of $A D$. At present, it is considered that the medial temporal lobe (including the entorhinal cortex, the side olfactory cortex, the side hippocampal cortex, and the hippocampus), the prefrontal lobe, and the precuneus are important areas related to episodic memory (12). It was found in the present study that ReHo values in the precuneus and medial temporal lobe were significantly lower than that in the control group. This is similar to previous research results $(13,14)$. Furthermore, Tau protein deposition and magnetic resonance results revealed that changes in atrophy degree is along the pathway of hippocampal atrophy (entorhinal cortex and hippocampus), and is consistent with the decline of early memory $(15,16)$. The medial temporal lobe may be the central area associated with episodic memory. When the patient has not yet developed into $A D$, time-series homogeneity of BOLD signals in the medial temporal lobe system is decreased (17-19); therefore, the medial temporal lobe can be used as a target to distinguish $\mathrm{MCl}$ patients from normal elderlies. In addition, ReHo values significantly decreased in some brain areas of the left inferior temporal gyrus and middle temporal gyrus suggesting that in addition to the medial temporal lobe, the outer portion of the left temporal lobe may also be closely correlated to episodic memory.

Episodic memory disorders are the outstanding manifestations of $A D$ patients; language barriers also exist. Difficulties in finding words and the mild decline in understanding ability occur in the early stage, and the abilities of reading and writing decline with the development of the disease. The results of a study indicated (20) that semantic dementia mainly involves the temporal lobe structure, especially the new cortex in the temporal lobe, which manifest semantic memory disorders, anomia, empty speech, and lack of rational words. This is consistent with the results of the present study, in which ReHo values decreased in the outer portion of the left temporal lobe suggesting that the temporal lobe structure contributes to abnormal brain function in patients with aMCl.

In addition, visual spatial structure dysfunction can occur during the early stage of $A D$. This visual spatial structure dysfunction is often associated with parietal occipital lobe lesions. We found that in addition to the medial temporal lobe system, ReHo value significantly decreased in the right occipital lobe and lingual gyrus suggesting the presence of resting-state brain functional abnormalities in multiple brain areas, which are associated 
with memory in aMCl patients. This provides a good starting point for subsequent brain functional connectivity.

It was also found in this study that, compared with the normal group, $\mathrm{ReHo}$ value of aMCI patients increased and concentrated mainly in the right frontal lobe, left fusiform gyrus, inferior frontal gyrus, superior temporal gyrus, and right thalamus. These areas are associated with the language center and visual word area, which are closely correlated with the working memory. The episodic memory of $A D$ patients tends to be severely damaged, while abstract thinking and computing power are often not affected during the early stage. Studies have shown that when aMCl patients implemented certain tasks, there were activation performances in the frontal lobe, fusiform gyrus, and other brain areas $(21,22)$. There may be some kind of compensatory mechanism in these areas in patients. Furthermore, aMCl patients in the early stage manifested with episodic memory impairment, while executive functioning was relatively well preserved, which was consistent with the result that the ReHo value increased in these related brain areas in this study. Moreover, this further confirms the compensation hypothesis. This compensatory mechanism would likely slow down the progression of the disease.

Other methods have also been used in the diagnosis of aMCI. Structural MRI can show that gray matter atrophy is particularly severe in patients with $\mathrm{MCl}$ that progresses to $A D$, compared with those whose condition is less severe $(23,24)$. Therefore, gray matter atrophy is effective for predicting whether $\mathrm{MCl}$ will deteriorate into $A D$, but only

\section{References}

1. Petersen RC, Doody R, Kurz A, Mohs RC, Morris JC, Rabins PV, et al. Current concepts in mild cognitive impairment. Arch Neurol 2001; 58: 1985-1992, doi: 10.1001/ archneur.58.12.1985.

2. Petersen RC. Mild cognitive impairment as a diagnostic entity. J Intern Med 2004; 256: 183-194, doi: 10.1111/j.13652796.2004.01388.x.

3. Vannini P, Almkvist O, Dierks T, Lehmann C, Wahlund LO. Reduced neuronal efficacy in progressive mild cognitive impairment: a prospective fMRI study on visuospatial processing. Psychiatry Res 2007; 156: 43-57, doi: 10.1016/ j.pscychresns.2007.02.003.

4. Qi D, Wang A, Chen Y, Chen K, Zhang S, Zhang J, et al. Default mode network connectivity and related white matter disruption in type 2 diabetes mellitus patients concurrent with amnestic mild cognitive impairment. Curr Alzheimer Res 2017; 14: 1238-1246, doi: 10.2174/156720501466617 0417113441.

5. Cai S, Chong T, Peng Y, Shen W, Li J, von Deneen KM, et al. Altered functional brain networks in amnestic mild cognitive impairment: a resting-state fMRI study. Brain Imaging Behav 2017; 11: 619-631, doi: 10.1007/s11682016-9539-0. when the disease has progressed to a certain extent, and patients are not at the early stage. Thus, it is not a good method for early detection of aMCl. Increased white matter abnormalities detected by diffusion tensor imaging have also been used to reflect aMCl $(25,26)$. However, results of different studies are inconsistent, which may be due to the disease stage, as single domain aMCl patients only have memory impairment, but multidomain aMCl patients have executive function impairment that is related to the frontal lobe (25). Many studies used $\mathrm{FMRI}$ on $\mathrm{aMCl}$ patients during brain activities, such as associative memory encoding and recognition task, when the brain is not at resting state. The results of such methods are probably different between aMCI subtypes, because whole-brain analysis showed that the active brain regions between multi-domain $\mathrm{aMCl}$ and single domain aMCl patients are different (27). Compared with these methods, ReHo evaluation in resting-state fMRI can help us understand the characteristics of the brain neural network and its functions at the resting state in aMCl patients, and thus identify early aMCl to provide treatment at the best time point for the disease.

In summary, an fMRI study was performed on aMCl patients with the application of magnetic resonance equipment. Understanding the characteristics of the brain neural network and its functions at the resting state in aMCl patients can not only help identify $\mathrm{aMCl}$ at the early stage but can also provide treatment for this disease at the best time window. In addition, it is also of great significance to have a thorough understanding of brain functions.
6. Liu D, Yan C, Ren J, Yao L, Kiviniemi VJ, Zang Y. Using coherence to measure regional homogeneity of resting-state FMRI signal. Front Syst Neurosci 2010; 4: 24.

7. Raichle ME, MacLeod AM, Snyder AZ, Powers WJ, Gusnard DA, Shulman GL. A default mode of brain function. Proc Natl Acad Sci USA 2001; 98: 676-682, doi: 10.1073/ pnas.98.2.676.

8. Raichle ME. Neuroscience. The brain's dark energy. Science 2006; 314: 1249-1250, doi: 10.1126/science. 1134405.

9. McKiernan KA, Kaufman JN, Kucera-Thompson J, Binder JR. A parametric manipulation of factors affecting taskinduced deactivation in functional neuroimaging. $J$ Cogn Neurosci 2003; 15: 394-408, doi: 10.1162/08989290332 1593117

10. Qi Z, Wu X, Wang Z, Zhang N, Dong H, Yao L, et al. Impairment and compensation coexist in amnestic $\mathrm{MCl}$ default mode network. Neuroimage 2010; 50: 48-55, doi: 10.1016/j.neuroimage.2009.12.025.

11. Sala-Llonch R, Bosch B, Arenaza-Urquijo EM, Rami L, Bargalló N, Junqué $C$, et al. Greater default-mode network abnormalities compared to high order visual processing systems in amnestic mild cognitive impairment: an integrated 
multi-modal MRI study. J Alzheimers Dis 2010; 22: 523-539, doi: 10.3233/JAD-2010-101038.

12. Budson AE, Price BH. Memory dysfunction. N Engl J Med 2005; 352: 692-699, doi: 10.1056/NEJMra041071.

13. He Y, Wang L, Zang Y, Tian L, Zhang X, Li K, et al. Regional coherence changes in the early stages of Alzheimer's disease: a combined structural and resting-state functional MRI study. Neuroimage 2007; 35: 488-500, doi: 10.1016/ j.neuroimage.2006.11.042.

14. Bai F, Zhang Z, Yu H, Shi Y, Yuan Y, Zhu W, et al. Defaultmode network activity distinguishes amnestic type mild cognitive impairment from healthy aging: a combined structural and resting-state functional MRI study. Neurosci Lett 2008; 438: 111-115, doi: 10.1016/j.neulet.2008. 04.021.

15. Thompson PM, Hayashi KM, de Zubicaray G, Janke AL, Rose SE, Semple J, et al. Dynamics of gray matter loss in Alzheimer's disease. J Neurosci 2003; 23: 994-1005, doi: 10.1523/JNEUROSCI.23-03-00994.2003.

16. Scahill RI, Schott JM, Stevens JM, Rossor MN, Fox NC. Mapping the evolution of regional atrophy in Alzheimer's disease: unbiased analysis of fluid-registered serial MRI. Proc Natl Acad Sci USA 2002; 99: 4703-4707, doi: 10.1073/ pnas.052587399.

17. Greicius MD, Srivastava G, Reiss AL, Menon V. Defaultmode network activity distinguishes Alzheimer's disease from healthy aging evidence from functional MRI. Proc Natl Acad Sci USA 2004: 101: 4637-4642.

18. Gómez-Isla T, Price JL, McKeel DW Jr, Morris JC, Growdon $\mathrm{JH}$, Hyman BT. Profound loss of layer entorhinal cortex neurons occurs in very mild Alzheimer's disease. J Neurosci 1996; 16: 4491-4500, doi: 10.1523/JNEUROSCI.16-1404491.1996.

19. Dickerson BC, Salat DH, Bates JF, Atiya M, Killiany RJ, Greve DN, et al. Medial temporal lobe function and structure in mild cognitive impairment. Ann Neurol 2004; 56: 27-35, doi: 10.1002/ana.20163.
20. Rohrer JD, Warren JD, Modat M, Ridgway GR, Douiri A, Rossor MN, et al. Patterns of cortical thinning in the language variants of frontotemporal lobar degeneration. Neurology 2009; 72: 1562-1569, doi: 10.1212/WNL.0b013e 3181a4124e.

21. Machulda MM, Ward HA, Borowski B, Gunter JL, Cha RH, O'Brien PC, et al. Comparison of memory fMRI response among normal, $\mathrm{MCl}$, and Alzheimer's patients. Neurology 2003; 61: 500-506, doi: 10.1212/01.WNL.0000079052. 01016.78 .

22. Yetkin FZ, Rosenberg RN, Weiner MF, Purdy PD, Cullum CM. FMRI of working memory in patients with mild cognitive impairment and probable Alzheimer's disease. Eur Radiol 2006; 16: 193-206, doi: 10.1007/s00330-005-2794-x.

23. Killiany RJ, Gomez-Isla T, Moss M, Kikinis R, Sandor T, Jolesz $F$, et al. Use of structural magnetic resonance imaging to predict who will get Alzheimer's disease. Ann Neurol 2000; 47: 430-439, doi: 10.1002/1531-8249(200004) 47:4<430::AID-ANA5 > 3.0.CO;2-I.

24. Visser PJ, Verhey FR, Hofman PA, Scheltens P, Jolles J. Medial temporal lobe atrophy predicts Alzheimer's disease in patients with minor cognitive impairment. J Neurol Neurosurg Psychiatry 2002; 72: 491-497.

25. Ibrahim I, Horacek J, Bartos A, Hajek M, Ripova D, Brunovsky $\mathrm{M}$, et al. Combination of voxel based morphometry and diffusion tensor imaging in patients with Alzheimer's disease. Neuro Endocrinol Lett 2009; 30: 39-45.

26. Agosta F, Pievani M, Sala S, Geroldi C, Galluzzi S, Frisoni $\mathrm{GB}$, et al. White matter damage in Alzheimer disease and its relationship to gray matter atrophy. Radiology 2011; 258: 853-863, doi: 10.1148/radiol.10101284.

27. van der Meulen M, Lederrey C, Rieger SW, van Assche M, Schwartz S, Vuilleumier $\mathrm{P}$, et al. Associative and semantic memory deficits in amnestic mild cognitive impairment as revealed by functional magnetic resonance imaging. Cogn Behav Neurol 2012; 25: 195-215, doi: 10.1097/WNN. 0b013e31827de67f. 